\title{
Impact of adding semaglutide in a person with type 1 diabetes: A case report
}

\author{
Sofra D* and Beer S \\ FMH diabétologie et endocrinologie, Cabinet médical, Lausanne, Switzerland
}

\begin{abstract}
Overweight woman with type 1 diabetes (T1D) treated with insulin pump and what else? Our understanding of type 1 diabetes and our capacity to standardize care as well as reduce the burden connected to the daily fluctuations of blood sugar remain largely unsatisfactory.

The daily life of a person with T1D shows that insulin therapy alone does not suffice to get the targeted glucose control in a majority of patients.

In the literature, data on the use of agonists of the receptor of Glucagon-like peptide-1 (AR GLP-1) in the patient with T1D are very few, but let us imagine that a positive impact can be expected. In particular, semaglutide has been studied a lot in the reduction of cardiovascular risk in persons with type 2 diabetes (T2D), but there is no data to date in the patient with T1D, neither on glycemic control, nor, or even less, on secondary complications.

This article exposes the clinical case of a woman with T1D, treated by insulin pump with then the addition of semaglutide. Using a flash type continuous glucose monitoring system, the monitoring parameters will be illustrated. The addition of semaglutide seems to have the ability to reduce the glucose variability, improve the glucose control and reduce weight in a patient with long time evolving T1D.
\end{abstract}

\begin{abstract}
Abbreviations: AR GLP-1: Agonist Receptor glucagon like peptide-1; BMI: Body Mass Index; CV: Coefficient of variation for glucose (\%); DBP: Dyastolic Blood Pressure; DR: Diabetic retinopathy; FPG: Fasting Plasma Glucose; GLP-1: Glucagon like peptide- 1; HbA1c: Glycated Haemoglobin; HRV: Heart rate variability; SBP: Systolic Blood Pressure; T1D: Type 1 diabetes; T2D: Type 2 diabetes.
\end{abstract}

\section{Introduction}

Type 1 diabetes is a disease characterised by the autoimmune destruction of the insulin producing pancreatic beta cells. Therefore, patients have to receive insulin every day in multiple injections or by continuous infusion with a pump, throughout their entire life [1]. But this treatment strategy doesn't allow a precise control of blood glucose. Hypoglycaemia, weight gain and glucose variability are often limiting factors to reaching the glucose targets as well as reducing the risk of diabetes related vascular complications. A particular attention has recently been given to glucose variability, defined as the fluctuation of blood glucose or other parameters of glucose homeostasis, such as glycated haemoglobin (HbA1c), on a given time period [2]. The intraday variability is the most reliable index, calculated with the coefficient of variation for glucose $(\% \mathrm{CV}=$ [standard deviation of the distribution of glucose around its average] / [blood glucose average]), with a threshold at $36 \%$ separating labile diabetes from stable diabetes [3]. The long term variability negative role, be it fasting glucose variations or HbAlc between 2 visits, is very well documented nowadays [4].

In physiological condition, glucose excursions are limited by the action of Glucagon-like peptide-1 (GLP-1). It is a hormone secreted by the L-cells of the small intestine and the distal colon exerting its effects through interaction with the GLP-1 receptor, expressed in the islets, the lungs, the hypothalamus, the stomach, the heart and the kidneys. GLP1 is part of the incretins group, it stimulates insulin secretion through the pancreatic beta cells, inhibits that of the glucagon through the pancreatic alpha cells, reduces hunger by impact on the hypothalamus as well as slowing down the gastric emptying.

The therapeutic class of the agonists of the receptor of GLP-1 (AR GLP-1) has now become one of the treatments of T2D, validated by the international recommendations [5,6]. They also allow a significant weight loss. However, their use in T1D is not approved, even though they may have interesting effects.

Adding AR GLP-1, traditionally used in T2D, to insulin in type 1 diabetes, was explored to improve glucose control, reduce glucose variability, decrease the insulin doses, and maintain or even lose weight [7]. It has been shown that adding liraglutide to insulin in persons with T1D over a 6 months period leads to an improvement of glucose control shown by the HbAlc [8].

Among the AR GLP-1, semaglutide, with a 94\% similarity to native GLP-1, is among the ones with a half-life extended by 1 week, which makes it appropriate for subcutaneous injection once a week. The main mechanism for its duration is the link to albumin, which causes a reduction of renal clearance and a protection against degradation by the DPP4 enzyme. The efficiency and safety of semaglutide have been reviewed in the SUSTAIN studies for T2D [9]. Treatment with semaglutide led to a steady improvement of the HbAlc as well as the

${ }^{\star}$ Correspondence to: Daniela Sofra, FMH diabétologie et endocrinologie, Cabinet medical, Avenue de la Gare 11, CH-1003 Lausanne, Switzerland, Tel: +41 (0)21312 61 01; Fax: +41 (0)21 31211 89, E-mail: daniela.sofra@svmed.ch

Key words: agonist receptor GLP-1, type 1 diabetes, glycaemic variability, semaglutide

Received: April 26, 2019; Accepted: May 22, 2019; Published: June 04, 2019 
bodyweight compared to the placebo and the active comparators. In addition, semaglutide showed a significant reduction of $26 \%$ of cardiovascular events [10]. This same study showed that treatment with semaglutide was connected to an increased risk of complications of diabetic retinopathy (DR) compared to standard treatment $(3,0 \%$ vs $1.8 \%$ ). In $>80 \%$ of cases, the patients already had DR lesions at the beginning of treatment.

Deeper analysis of these data, presented by Vilsboll and colleagues, allowed to establish a relation between the too swift correction of chronic hyperglycaemia with the initial aggravation of diabetic retinopathy, even though the long term improvement of glucose control reduces the risk [11]. Nevertheless, treatment with AR GLP-1 remains restricted to $\mathrm{T} 2 \mathrm{D}$.

\section{Clinical case}

Overweight Caucasian woman, born in 1987, in good general health, with T1D since 1999. The therapeutic follow-up, after paediatrics, started in 2011. Despite the patient being very involved and regularly monitoring her blood glucose, realising her boluses, doing her best to manage daily life and its uncertainties, glucose control remained poor as shown by the different measures of the HbAlc ranging from $8.5 \%$ to $10 \%$ (Table 1 ). In order to improve the glucose control, in June 2014, we went from a basal/bolus type insulin regimen to a patch type insulin pump. The improvements at the time seemed promising, since we were able to reduce the basal insulin dosis by almost $50 \%$, improve the weight and obtain a decrease of the HbAlc from $11.5 \%$ to $8.9 \%$. Yet in the 5 following years, the improvements remained limited with an average glycaemia of $13.5 \mathrm{mmol} / \mathrm{L}$. We added a flash type continuous glucose monitoring system in order to be able to better monitor and manage the glucose fluctuations. The dietary management was revised with the help of a dietician to better evaluate the bolus/meal ratio.

The main difficulty we encounter was to reduce glucose variability, limit weight gain, improve the $\mathrm{HbAlc}$, flatten the morning peak without increasing the occurrence of hypoglycaemia. The continuous glycaemia recording made in January 2019, in Figure 1, clearly illustrates the difficulties encountered. The patient complained mostly about being exhausted, gaining weight and being irritable. She described herself as being exhausted because she believed that the diabetes related stress had a negative impact on her love life, her physical activities, her professional and social life.
Before intensifying the treatment, we explored all the possible micro and macro vascular complications (Table 2). The slight increase of the average heart rate should be noted, with 94 beat/min (recorded by 24 hour monitoring) as well as the presence of severe background DR and slight macular degeneration in the left eye.

At the beginning of January, the decision is made to introduce a long acting AR GLP-1. So as not to overburden daily life, knowing that the patient has a tendency to an increased heart rate (studies show a significant rise with liraglutide [12]), the choice goes to a weekly injection of semaglutide.

The informed consent was obtained so that the data could be presented in the case.

\section{Results}

We made the assumption that adding semaglutide to the usual insulin pump treatment, with this woman with 20 years evolution of diabetes, could improve the glucose profile, reduce the \%CV, while limiting the gain weight. Semaglutide was introduced as planned in stages of $0.25 \mathrm{mg} /$ week for 4 weeks and then increased to $0.5 \mathrm{mg} /$ week. Gastrointestinal disorders were reported in titration phase, mostly nausea, yet moderate ones that did not require any treatment and were not hindering the increment of the therapeutic dose to $0.5 \mathrm{mg}$. After the therapeutic change, the patient notices that she feels better overall, now "I've got time to live" and that, for the very first time in her life with diabetes, she obtained an HbAlc in the target (Figure 1).

The improvement is clear on several levels: glucose levels in absolute terms, the $\% \mathrm{CV}$, the weight, the fasting glucose value, the $\mathrm{HbAlc}$ as well as the efforts the patient has to provide to maintain the values she has reached.

We manage to reduce the insulin dosage in the pump's basal rate by $-5.2 \mathrm{UI} / 24 \mathrm{~h}$, quickly after the introduction of semaglutide, before the weight loss. Thereafter, the patient reduced her weight by $-5 \mathrm{~kg}$ (= reduction of $8 \%$ of bodyweight), thereby reaching a BMI within the normal limits and we recorded an improvement of the HbAlc of $-1.9 \%$ (Figure 2). The analysis of the continuous glucose recording 3 months after therapeutic change shows the decrease of average glucose level $(8.3 \mathrm{mmol} / \mathrm{l}$ vs $10.8 \mathrm{mmol} / \mathrm{l})$, the improvement of glucose variability shown by the increase of percentage of glycaemia in the target, defined between 3.9 and $8.9 \mathrm{mmol} / \mathrm{l}$ ( $63 \%$ vs $38 \%$ ), yet with an increment of the percentage of hypoglycaemia ( $6 \%$ vs. $2 \%)$.

Table 1. Diabetes patient's medication changes over time

\begin{tabular}{|c|c|c|c|}
\hline Date & Medication change & HbA1c (\%) at time of change & - \\
\hline 1999 Diabetes diagnostic & Basal/Bolus & 9.3 \\
\hline 2011 Start of visits & Insulin Detemir 52 U/day & - \\
\hline 2012 & Insulin Detemir 26 UI + NPH 28 U/day & 28.9 \\
\hline 2013 & Insulin Degludec 40 U/day & 11.5 & 27.5 \\
\hline 2014 & Insulin pump basal 25,5 U/day & 8.9 \\
\hline 2105 to 2019 & Insulin pump basal \pm 27 U/day & 8.7 to 9.4 \\
\hline
\end{tabular}

HbA1c: Glycated Haemoglobin; BMI: Body Mass Index

Table 2. Exploration of potential complications

\begin{tabular}{|c|c|c|c|}
\hline & Date & \multicolumn{2}{|c|}{ Results } \\
\hline \multirow{2}{*}{ Eyes } & 21.12 .2018 & \multicolumn{2}{|c|}{ Non-prolifervative bilateral severe DR and left eye maculopathy } \\
\hline \multirow{2}{*}{ Heart } & 15.12 .2018 & HRV 94/min & SBP $130 \mathrm{mmHg}$ \\
\cline { 2 - 4 } & 09.04 .2019 & HRV 80/min & SBP $120 \mathrm{mmHg}$ \\
\hline \multirow{2}{*}{ Kidneys } & 31.10 .2018 & Urinary Albumin $/$ Creatinine $2.8 \mathrm{mg} / \mathrm{ml}$ \\
\cline { 2 - 4 } & 09.04 .2019 & Urinary Albumin $/$ Creatinine $2.0 \mathrm{mg} / \mathrm{ml}$ \\
\hline
\end{tabular}

DR: Diabetic retinopathy; HRV: Heart rate variability; SBP: Systolic Blood Pressure; DBP: Dyastolic Blood Pressure 
The analysis of the coefficient of variation, made easy by the continuous glucose recordings, showed that, compared to the threshold of $36 \%$, the Selmaglutide quickly allowed to come close to the desired therapeutic goal compared to the insulin treatment alone (Figure 3). Furthermore, to illustrate the deep changes induced in daily life by the addition of the AR GLP-1, Figure 4 shows two days of continuous glucose recordings with and without semaglutide.

It should be noted that, in this clinical case, we do not have the data concerning the C-peptide. However, based on the literature, this may not have brought to different conclusions as to the necessity to reduce the insulin dose following the addition of AR GLP-1. Indeed, based on Kielgast study [13] on the impact of a 4 weeks treatment with liraglutide in type 1 diabetes patients with and without residual beta cell function, according to C-peptide dosage, it has been established that they all needed an insulin reduction.

\section{Discussion}

The clinical case described here strengthens the hypothesis, already demonstrated with type 2 diabetes patients, that with some people with diabetes, the plasma concentrations of glucagon can be inappropriately high, which translates clinically in a fasting and nighttime hyperglycaemia [14]. Therefore, drugs like AR GLP-1 are unique because they target the glucagon pathway. In our clinical case, it seems that improving the glucose control with semaglutide (as shown if figure 4) is also possible in a person with T1D, during the night and morning

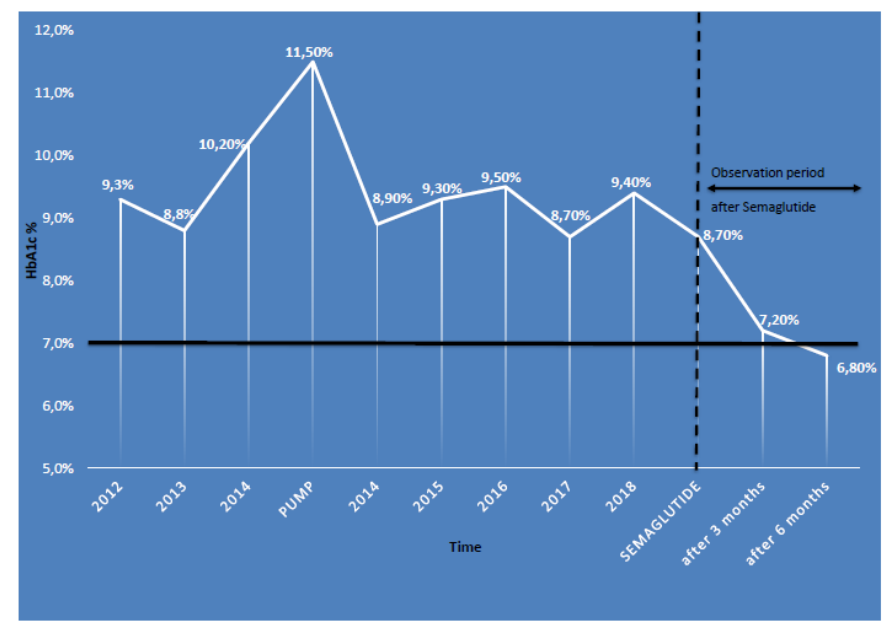

Figure 1. A schematic evolution of the $\mathrm{HbA} 1 \mathrm{c}(\%)$ at time of change

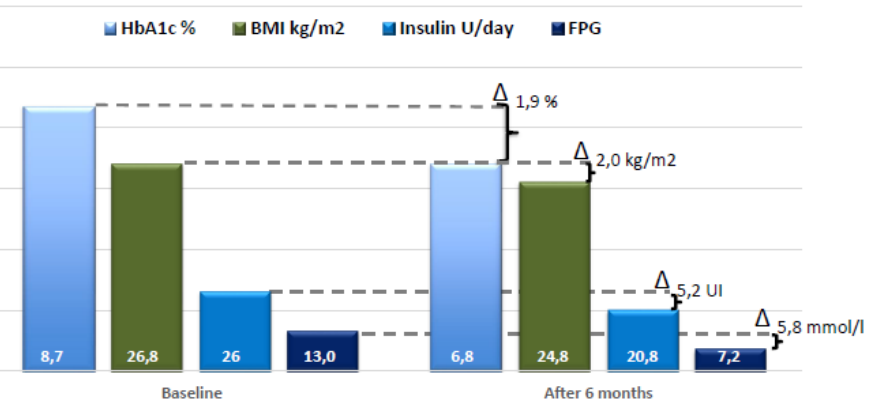

Figure 2. Evaluation before and after semaglutide

HbA1c: Glycated Haemoglobin; BMI: Body Mass Index; FPG: Fasting Plasma Glucose; $\Delta:$ Mean Change from baseline

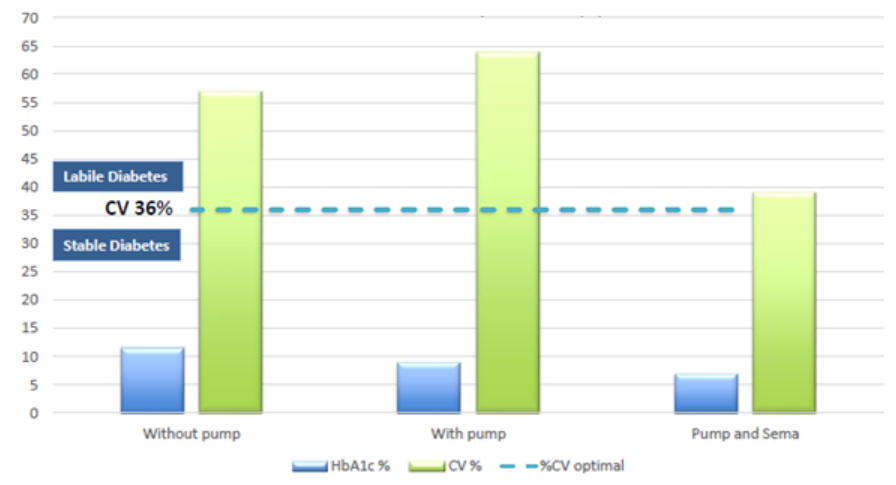

Figure 3. Glucose variability coefficient (\%)

HbA1c: Glycated Haemoglobin; CV: Coefficient of variation for glucose

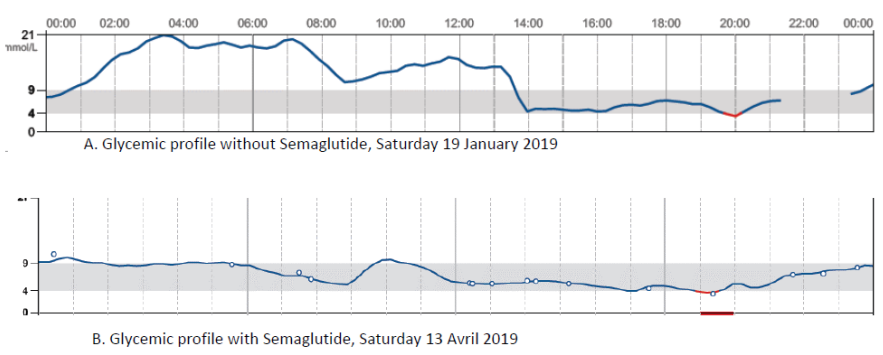

Figure 4. Illustration of two days of continous blood glucose monitoring (free style) in the patient

A. without semaglutide B. with semaglutide

glycaemia, by fighting hyperglucagonemia, as recent studies also seem to show [15]. This is probably owed to several mechanisms working in synergy. Increasing the gastric emptying time with AR GLP-1, as demonstrated by Horowitz, allows a decreasing of postprandial glucose, thereby reducing the speed at which glucose appears in the blood flow after the meal [16]. The weight loss following the semaglutide injections also plays a major role in improving the glucose profile in overweight patients. In the clinical case described here, in addition to the insulin pump, the treatment with AR GLP-1 caused an $8 \%$ reduction of the baseline bodyweight. In this woman with type 1 diabetes, semaglutide reduced the average glucose level (10.8 vs $8.1 \mathrm{mmol} / \mathrm{l})$ and improved the glucose variability, maybe due to its effect on glucagon secretion. Furthermore, the studies did show how much semaglutide reduces the energy intake compared to the placebo. During the consultation, the patient did describe very well the suppression of appetite that enabled her to have a better control of her diet and less food cravings. The addition of an AR-GLP1 as a supplement to insulin is very interesting, with a decrease of $1.9 \%$ of HbAlc in this clinical case.

A 2016 study of Ilkowitz [17] about the addition of liraglutide to an insulin pump treatment in 15 type 1 diabetes patients, showed a decrease of the dose of insulin as well as a decrease of glucose excursions, as it was also the case with our patient.

However, we have not explored the perception of hypoglycaemia in detail. Up to this day, 4 months after beginning the treatment with semaglutide, hypoglycaemias don't seem to decrease either in number or in frequency. The degree of hypoglycaemia remaining limited in this patient, we could not investigate whether adding AR GLP-1 may enable a recovery of sensitivity to hypoglycaemia. Indeed, a study recently conducted with exenatide for 6 weeks concluded that this AR GLP-1 could not improve the perception of hypoglycaemia [18]. When questioned about the quality of life, the patient finds that, compared 
to her own life before the change of therapy, the results are positive. Among other things, she notes a better physical and psychological health than a year before. No limitation in daily activities, nor in practising any physical activity. Indeed, the ADJUNCT ONE [7] study about the safety of liraglutide in addition to insulin therapy in persons with T1D, looked into the question of the quality of life. They had shown that patients who received liraglutide were overall satisfied, with a score statistically higher in the intervention group than in the placebo group. Our experience through this clinical case seem to corroborate this, even though it wasn't tested with a validated score.

These observations lead to extend the reflection to the chronic patient counselling. Our role consists in providing the best treatments to excel not only in glucose control, but also in terms of quality of life, reduction of complications. An adjuvant treatment with AR-GLP1 can reduce the glucose variability, and therefore reduce the overall risks. The significant proportion of persons with T1D who don't meet the desired glucose targets, the increment of hypoglycaemia in intensive insulin therapy and the increasing of obesity highlight the need for individualised clinical care in T1D just like in T2D, beyond insulin therapy and efficient technology.

\section{Conclusion}

Through the clinical situation which we presented, we help complete the few data available on the use of AR GLP-1 in persons with type 1 diabetes. The evaluation of these new therapies - efficient in type 2 diabetes - as a supplement to insulin therapy, represent a promising strategy.

Given the positive impact on the cardiovascular and renal levels, observed in the LEADER and SUSTAIN studies in persons with type 2 diabetes with a high risk of vascular complications that are also observed in persons with T1D, this therapeutic regimen can considerably improve the quality of life and help reach glucose targets that had been impossible to reach so far. However, it should be stressed that prescribing the AR GLP-1 does not respect the approved guidelines for T1D.

Waiting for further conclusive data to support this strategy, this case report adds a stone to the building. It shows the positive impact of adding semaglutide to an insulin treatment in an overweight person, with a T1D evolving for over 20 years, with insufficient glucose control.

\section{Acknowledgements}

To the patient for consenting to share her data to realise this case report.

\section{Declaration of interest}

The authors hereby declare having no conflicts of interest in connection with the writing of this manuscript, yet declares D. Sofra receiving personal fees from Novo Nordisk, Merck Sharp \& Dohme,
Sanofi and Pronokalgroup as speaker in different conferences, seminars and training courses.

\section{References}

1. Shields BM, McDonald TJ, Oram R (2018) C-peptide decline in type 1 diabetes has two phases: an initial exponential fall and a subsequent stable phase. Diabetes Care 41 : 1486-1492. [Crossref]

2. Monnier L (2018) The application of simple metrics in the assessment of glycaemic variability. Diabetes Metab 44: 313-319. [Crossref]

3. Monnier L, Colette C, Wojtusciszyn A, Dejager S, Renard E, et al. (2017) Toward defining the threshold between low and high glucose variability in diabetes. Diabetes Care 40: 832-838. [Crossref]

4. Ceriello A (2019) Glycaemic variability in diabetes: clinical and therapeutic implications. Lancet Diabetes Endocrinol 7: 221-230. [Crossref]

5. [No authors listed] (2019) Introduction: Standards of medical care in diabetes-2019. Diabetes Care 42: S1-S2. [Crossref]

6. Aaboe, K, Krarup T (2008) GLP-1: physiological effects and potential therapeutic applications. Diabetes Obes Metab 10: 994-1003.

7. Mathieu C, Zinman B (2016) Efficacy and safety of liraglutide added to insulin treatment in type 1 diabetes: The ADJUNCT ONE treat-to-target randomized trial. Diabetes Care 39: 1702-1710. [Crossref]

8. Varanasi A, Bellini N, Rawal D, Vora M, Makdissi A, et al. (2011) Liraglutide as additional treatment for type 1 diabetes. Eur J Endocrinol 165: 77-84. [Crossref]

9. R Aroda V, Ahmann A, Cariou B, Chow F, Davies MJ, et al. (2019) Comparative efficacy, safety, and cardiovascular outcomes with once-weekly subcutaneous semaglutide in the treatment of type 2 diabetes: Insights from the SUSTAIN 1-7 trials. Diabetes Metab 4: S1262-3636. [Crossref]

10. Marso SP, Bain SC, Consoli A, Eliaschewitz FG, Jodar E, et al. (2016) Semaglutide and cardiovascular outcomes in patients with type 2 diabetes. N Engl J Med 375: 18341844. [Crossref]

11. Vilsbøll T, Bain SC, Leiter LA (2018) Semaglutide, reduction in glycated haemoglobin and the risk of diabetic retinopathy. Diabetes Obes Metab 20: 889-897. [Crossref]

12. Kumarathurai P, Anholm C, Larsen BS, Olsen RH, Madsbad S, et al. (2017) Effects of liraglutide on heart rate and heart rate variability: a randomized, double-blind, placebocontrolled crossover study. Diabetes Care 40: 117-124. [Crossref]

13. Kielgast U, Krarup T, Holst JJ, Madsbad S (2011) Four weeks of treatment with liraglutide reduces insulin dose without loss of glycemic control in type 1 diabetic patients with and without residual B-Cell function. Diabetes Care 34: 1463-1468

14. D'Alessio D (2011) The role of dysregulated glucagon secretion in type 2 diabetes. Diabetes Obes Metab 1: 126-132. [Crossref]

15. Fredheim S, Andersen ML, Porksen S (2015) The influence of glucagon on postprandial hyperglycaemia in children 5 years after onset of type 1 diabetes. Diabetologia 58: 828834. [Crossref]

16. Marathe C, Rayner C, Wu T, Jones K, Horowitz M (2018) Gastric emptying and the personalized management of type 1 diabetes. J Clin Endocrinol Metab 103: 3503-3506. [Crossref]

17. Ilkowitz JT, Katikaneni R, Cantwell M, Ramchandani N, Heptulla RA (2016) Adjuvan liraglutide and insulin versus insulin monotherapy in the closed-loop system in type 1 diabetes: A randomized open-labeled crossover design trial. J Diabetes Sci Technol 10 1108-1114. [Crossref]

18. Van Meijel L, Rooijackers H, Tack C, De Galan BE (2019) Effect of the GLP-1 receptor agonist exenatide on impaired awareness of hypoglycemia in type 1 diabetes; a randomized controlled trial. J Clin Endocrinol Metab 8: 87. [Crossref]

Copyright: (2019 Sofra D. This is an open-access article distributed under the terms of the Creative Commons Attribution License, which permits unrestricted use, distribution, and reproduction in any medium, provided the original author and source are credited. 